\title{
Measurement of Density of Fe-Co Alloys Using Electrostatic Levitation
}

\author{
JONGHYUN LEE, JUSTIN E. RODRIGUEZ, ROBERT W. HYERS, \\ and DOUGLAS M. MATSON
}

\begin{abstract}
The density of a series of five different iron-cobalt alloys was measured using electrostatic levitator processing. Each sample was processed through multiple thermal cycles and the liquid density was measured, while the superheated sample was cooled down to its undercooled state. The volume of the sample was estimated by analyzing captured high-speed video data of the projected shape of the sample. The mass change during the melt cycle was also tracked using Langmuir's equation of mass evaporation. The density was then calculated as a function of temperature based on these measurements of volume and mass. Density values obtained showed higher precision than existing data from the literature obtained using a variety of different techniques, although the accuracy was consistent.
\end{abstract}

DOI: $10.1007 / \mathrm{s} 11663-015-0434-7$

(C) The Minerals, Metals \& Materials Society and ASM International 2015

\section{INTRODUCTION}

THE processing of metals and alloys frequently involves melting and solidification. The first step to understand and control these processes is to accurately measure material properties for metals and alloys in their liquid state. For example, accurate prediction of convection and heat transfer during a casting process is often of critical importance in order to control a casting process and to improve the quality of cast products. The accurate prediction of dynamic events which occur during liquid metal processing is a challenging task due to some technical difficulties encountered at high temperatures. Simulations using either analytical or numerical approaches require accurate material properties of liquid metals, such as density, viscosity, and surface tension. The availability of accurate density data is crucial as it must be known in order to simplify the procedures needed to measure both viscosity and surface tension. Therefore, there have been a number of efforts to increase the accuracy and precision of density measurements over a wide range of temperatures. Several traditional techniques for the density measurement include dilatometric, ${ }^{[1,2]}$ pycnometric, ${ }^{[3]}$ aerometric, ${ }^{[4]}$ radiographic ${ }^{[5]}$ Archimedean, ${ }^{[6]}$ maximum bubble pressure (MBP), ${ }^{[7,8]}$ and liquid drop ${ }^{[9]}$ methods. The accuracy and applicability of these methods is limited due to the chemical instability of specimens and test apparatus.

JONGHYUN LEE, Research Assistant Professor, and ROBERT W. HYERS, Professor, are with the Mechanical and Industrial Engineering Department, University of Massachusetts, 160 Governors Drive, Amherst, MA 01003. JUSTIN E. RODRIGUEZ, Research Associate, and DOUGLAS M. MATSON, Associate Professor, are with the Mechanical Engineering Department, Tufts University, 200 College Avenue, Medford, MA 02155. Contact e-mail: douglas. matson@tufts.edu

Manuscript submitted May 9, 2014.

Article published online August 14, 2015.
In recent decades, containerless processing has emerged as an effective tool for measuring thermophysical properties of molten metals at high temperatures. By innovatively removing containers, containerless processing essentially eliminates the potential for chemical reaction between the specimen and testing apparatus. The advantages of containerless processing compared to the conventional methods are the lower risk of sample contamination, the wider range of processing temperatures, and the accessibility to metastable states and phases. There are two widely used non-contact methods: electromagnetic levitation (EML) and electrostatic (ESL) levitation.

EML removes the necessity of a container by floating a conductive sample of 5 to $8 \mathrm{~mm}$ diameter using Lorentz forces generated by alternating currents running through water-cooled copper tube coils. The upper and lower parts of the coil are counter-wound to generate a quadrupole electromagnetic field in which the sample is stabilized. Normally, the lower EML coils must have more turns to generate upward resultant forces equivalent to the weight of the sample. The resistive heating of the sample is achieved at the same time by the induced eddy currents in the sample mainly near the surface in a thin layer characterized by the skin depth. In the course of processing, the surface temperature of the sample is monitored by an optical pyrometer which converts the emissive power emitted from the sample surface into a temperature signal based on the knowledge of the emissivity of the sample over a narrow spectral wavelength. The oxidation and mass evaporation of the melt are significantly reduced by surrounding the sample with an inert gas at a pressure of $\sim 0.5$ to 0.7 bar. During a typical melt cycle, the shape of the sample is captured with a high-speed camera. In the lab, an EML sample deforms into an obloid since gravity pulls the sample down through the quadruple electromagnetic field. This deformation must be taken into 
account during volume estimation, and minor surface oscillations will thus induce some error-mainly influencing precision without influencing measurement accuracy. Tracking the mass and volume yields the density as a function of time and temperature. Examples of the results of this type of density measurement of molten materials using EML can be found elsewhere. ${ }^{[10-12]}$

In contrast, ESL utilizes repulsive Coulomb forces to cancel gravity. The size and mass of the sample generally are 2 to $3 \mathrm{~mm}$ in diameter and $40 \mathrm{mg}$, respectively. The surface of the sample is charged negatively with electrons discharged from a deuterium arc lamp, while the bottom electrode is also charged negatively such that the sample is repelled by the bottom electrode. The stability of the sample is ensured by two orthogonal pairs of electrodes on the horizontal plane controlled by signals from a positioning laser couple which detects the horizontal location of the levitated sample through back illumination. After levitation and stabilization, a heating laser is applied to the sample. As in the case of EML, an optical pyrometer is used to monitor the surface temperature of the sample, and the projected area of the sample is again captured by a high-speed camera. ESL processing must be done in a vacuum chamber to prevent arcing between the vertical electrodes due to their substantial potential difference $(\sim 14 \mathrm{kV})$.

Comparing EML and ESL techniques, it is apparent that during EML processing sample deformation complicates the ability to track sample volume. Since the processing pressure and sample vapor pressures are of similar magnitude and given the large sample mass, evaporation is often negligible. For ESL samples, the small sample size results in minimal deviation from spherical shape allowing high confidence in volume estimation; however, due to the possibility of sample evaporation, the mass must be carefully monitored. Vacuum processing of high-temperature materials may involve mass evaporation which becomes more significant when the sample has a high vapor pressure - for instance for cobalt and iron. Therefore, mass evaporation must be tracked accurately as a function of time and temperature throughout each thermal cycle. Estimation of mass evaporation during ESL test is not a trivial task due to several reasons including small sample sizes, uncertainties in thermophysical properties, and complications induced by chemical and thermodynamic interactions among constituents. This paper describes the measurement of the density of iron-cobalt alloys using an ESL technique in order to demonstrate the superior precision in thermophysical property measurement for alloy compositions with a high vapor pressure. In previous research ${ }^{[13]}$ tracking mass evaporation during a thermal cycle for samples of $\mathrm{Fe}_{50} \mathrm{Co}_{50}$ was demonstrated successfully. The final mass and final composition shift were predicted with errors less than 5 pct and 4 pct, respectively. In the current work, the same method was applied to track the mass change for five different $\mathrm{Fe}-\mathrm{Co}$ compositions during density measurement. The density results obtained using ESL were compared with existing data measured using EML and by the MBP method to compare the precision of each technique during density measurement of molten alloys with a high vapor pressure.

\section{EXPERIMENTAL DETAILS}

$4 \mathrm{~N} \mathrm{Fe}$ and $3.5 \mathrm{~N}$ Co wires were cut and filed to meet specific compositions, and they were arc-melted in an argon atmosphere to form alloys. For the case of samples tested, mass loss during arc melting was routinely as low as $0.1 \mathrm{pct}$ and the resulting composition shift was less than 0.011 at. pct. The tested compositions were pure $\mathrm{Fe}, \mathrm{Fe}_{70} \mathrm{Co}_{30}, \mathrm{Fe}_{60} \mathrm{Co}_{40}, \mathrm{Fe}_{50} \mathrm{Co}_{50}$, and pure Co in atomic percentage. The ESL facility located at the NASA Marshall Space Flight Center (MSFC) in Huntsville, AL, was used for the levitation experiments. Figure 1 describes the facility used. Before measurement, the vacuum chamber was evacuated down to $\sim 10^{-6}$ torr using a turbo molecular pump. A machined WC-Co calibration sphere of $2.2 \mathrm{~mm}$ in diameter was loaded, and its projected area was captured to be utilized as a reference value for subsequent volume calculation. The camera was focused such that the radial color gradient across the edge of the projected sample image was between 4 and 8 pixels. The calibration sphere was re-evaluated post-test to ensure that no changes had occurred in the optical path. During the density analysis process, the midpoint of color gradient across the edge of the sample was found, and the encompassed area was taken to be the projected area of the sample.

After a solid sample was levitated and stabilized as shown in Figure 1(b), a $200 \mathrm{~W}$ YAG laser was applied to heat the sample. During thermal cycles, the projected shape of the sample is captured by a Redlake high-speed camera model number Y4-S1 with an image size of $512 \times 512$ pixels at a rate of 25 frames per second and a corresponding resolution of $\sim 1.5 \mathrm{mi}-$ crons/pixel. An example of captured sample image is given in Figure 1(c). Meanwhile, the temperature of the sample was tracked using an optical pyrometer manufactured by Mikron Infrared Inc. model number IMPAC IGA 140 operating at a characteristic wavelength of 1.4 to $1.8 \mu \mathrm{m}$ with an acquisition rate of $1.67 \mathrm{~Hz}$. Figure 2 shows an example of a representative temperature-time profile. The sample was heated from the solid state (1), became molten (2), and was superheated (3) until the adequate superheating (4) was achieved. As soon as the heating laser is turned off, the sample cooled (5) down freely to its maximum undercooling temperature (6). For the sample in Figure 2, the amount of undercooling increased from $53.0 \mathrm{~K}$ to $72.8 \mathrm{~K}$ as the number of melt cycles accumulated. The undercooling of the other samples, not shown, ranged from 27.4 to $166.3 \mathrm{~K}$ and also showed the same trend where the undercooling increased as test time progressed. The variation in undercooling is attributed to the changes in the level of impurity compositions over time. The main impurities present in the sample are oxides which float on the surface of melt and possibly serve as preferred heterogeneous nucleation sites. Superheating the sample helps 


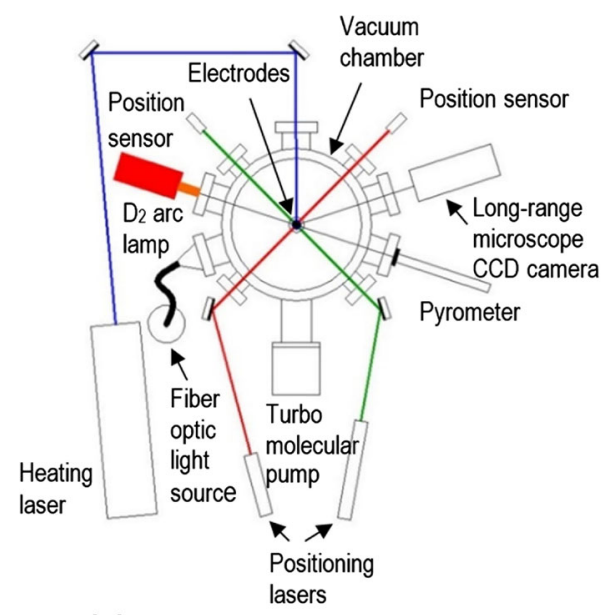

(a) A schematic of NASA MSFC ESL

Fig. 1-The ESL facility located at NASA MSFC.

decompose some of these oxides such that the amount of impurities generally reduces, and thus the sample exhibits deeper undercooling as the number of melt cycles increases. After reaching its maximum undercooling, the sample solidified rapidly (7) rejecting the remaining latent heat by radiation during recalescence. Often, a two-step rapid solidification path could be observed as seen in Figure 2. The low-temperature $\gamma$-phase-to- $\alpha$-phase transformation causes significant sample instability, and thus, before the solidified sample is allowed to cool down to below this temperature, it is prudent to turn the heating laser back on (8) to repeat the melt cycle.

The vapor pressure of iron and cobalt is relatively high, and thus noticeable mass evaporation was observed during ESL processing. An Fe-Co ESL sample that has undergone a single melt cycle loses material on the order of $\sim 1$ to $2 \mathrm{wt}$ pct due to mass evaporation. The sample in Figure 2 lost 4.75 pct of its mass over the entire multi-cycle duration of testing. The evaporated material is deposited both on the electrodes and on the observation windows. Dust deposited on the electrodes hinders the stable levitation of the sample by distorting the electrostatic field. Dust deposited on the chamber windows weakens the magnitude of the observed emissive power from the surface of the sample. This transmissivity shift leads to the gradual underestimation of the surface temperature with time. Therefore, the electrodes were cleaned and the observation windows were replaced after testing every 2 to 3 samples.

\section{DATA ANALYSIS}

The first step in data analysis involves recalibrating the pyrometer for any transmissivity shift. The apparent temperature data can easily be corrected from an evaluation of sample emissivity at the alloy melting point. Interpolating corrected temperature data at the video data frame rate allowed for a correlation of temperature and sample volume. Each video frame was

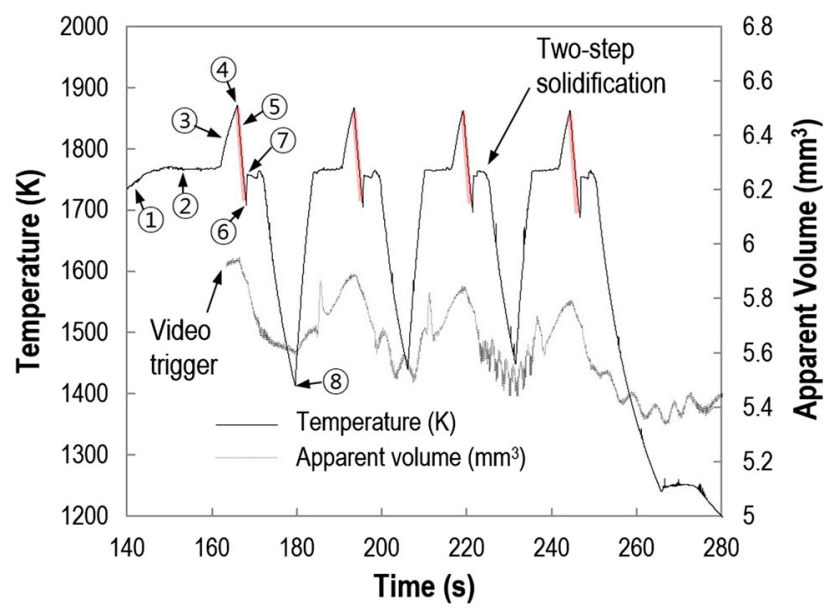

Fig. 2-An example of multiple melt cycles of one $\mathrm{Fe}_{70} \mathrm{Co}_{30}$ sample.

processed to count the number of pixels contained in the projected area of the sample. Based on the calibration sphere data, the diameter and volume of the tested sample for each frame were then estimated. To verify the sphericity of the sample, the ratio of the minor axis to the major axis was calculated from each frame of the video data for samples of $\mathrm{Fe}_{70} \mathrm{Co}_{30}, \mathrm{Fe}_{60} \mathrm{Co}_{40}$, and $\mathrm{Fe}_{50} \mathrm{Co}_{50}$. The results, given in Table I, indicate that the samples are nearly perfect spheres.

Several interesting features are observed in Figure 2 which shows a comparison of temperature and apparent volume as a function of time. Upon melting, a sharp increase in apparent volume was observed and can be seen as a temperature spike midway through the melt plateau. This sudden expansion and subsequent relaxation during melting is attributed to the coalescence of the central solidification void. Note that there is the large fluctuation in apparent volume for times when the sample was solid; this can be attributed to off-axis rotation of a non-spherical shape sample after solidification. Both frequency and amplitude vary based on cycle-specific sphericity and rotation rate following each thermal cycle. It can also be noticed that the apparent volume decreased as the number of melt cycles increased due to the cumulative effect of mass evaporation. Note that evaporation is a strong function of temperature and thus time at temperature during different parts of a single run becomes important.

The total mass loss, $m_{t}$, at time $t$ during a melt cycle can be expressed as Reference 13

$$
m_{t}=\sum_{i=1}^{n}\left[\int_{t_{0}}^{t} \frac{\alpha_{i} A_{i} a_{i}\left(P_{\mathrm{v}, i}-P_{\mathrm{ref}}\right)}{\sqrt{2 \pi M_{i} R T}} \mathrm{~d} t^{*}\right],
$$

where, for component $i, A_{i}$ is the effective surface area, $a_{i}$ is the activity, $P_{\mathrm{v}, i}$ is the vapor pressure, $P_{\mathrm{ref}}$ is the reference pressure, $M_{i}$ is the molecular weight, $R$ is the gas constant, and $T$ is the absolute temperature. The correction factor $\alpha_{i}$ indicates the degree of deviation from the ideal mass evaporation, and it takes the value of unity for the case of ESL vacuum evaporation where the evaporated atoms or molecules are assumed to never return to the bulk. 
Table I. Results of the Sphericity Analysis

\begin{tabular}{lccc}
\hline Composition & Averaged $d_{\text {minor }} / d_{\text {major }}$ & Standard Deviation & Number of Frames \\
\hline $\mathrm{Fe}_{70} \mathrm{Co}_{30}$ & 1.000376 & 0.005724 & 4500 \\
$\mathrm{Fe}_{60} \mathrm{Co}_{40}$ & 1.005182 & 0.005706 & 3001 \\
$\mathrm{Fe}_{50} \mathrm{Co}_{50}$ & 1.001674 & 0.007262 & 4000 \\
\hline
\end{tabular}

$d_{\text {minor }}$ is the length sample's minor axis, $d_{\text {major }}$ is the length of the sample's major axis.

There are three more factors to be considered when the mass evaporation of alloys is dealt with: surface segregation, solute activity, and changes to final composition due to differential evaporation of solute and solvent. ${ }^{[13]}$ The surface segregation is a measure of the difference between the composition in the bulk and the composition on the surface. This is manifested as an effective surface area for each constituent. For the case of iron-cobalt alloys, there is little difference in surface tension for both components at a given temperature. For example, the difference in surface tension of iron and cobalt at the melting temperature of $\mathrm{Fe}_{50} \mathrm{Co}_{50}$ is approximately $3 \mathrm{pct}^{[14]}$ which indicates that preferential segregation of one component can be assumed to be negligible - considering the uncertainties in other thermophysical data. Segregation can also be manifested as a change in composition between top and bottom of a sample due to preferential sedimentation of the denser component. EDS was performed using a Zeiss LEO 1530VP SEM equipped with an Si(Li)-EDX detector by Oxford Instruments and INCA software at DLR-Köln at selected locations for a nominal $\mathrm{Fe}_{50} \mathrm{Co}_{50}$ sample post-testing, and the results show no discernible segregation or sedimentation to the detection limits of the hardware.

The activity of iron and cobalt in each alloy was calculated using commercial thermophysical software, ThermoCalc ${ }^{\mathrm{TM}}$, over a full wide range of temperatures. The calculated activity of iron-cobalt alloys at $1750 \mathrm{~K}$ $\left(1476.85^{\circ} \mathrm{C}\right)$ as a function of composition is plotted in Figure 3(a). One can notice slight negative deviation from ideal behavior corresponding to a value of $\Omega=-8.182 \mathrm{~kJ}$ when modeling the deviation from ideality using a regular solution model. The corresponding change in activity of each of the constituents in the $\mathrm{Fe}_{50} \mathrm{Co}_{50}$ alloy is plotted in Figure 3(b) as a function of temperature. The activity of cobalt is slightly higher at lower temperatures $\left[<\sim 1200 \mathrm{~K}\left(926.85^{\circ} \mathrm{C}\right)\right]$ and slightly lower at higher temperatures. Since the rate of mass evaporation of iron is up to 17 times larger than that of cobalt, the final mole fraction of iron decreases if significant mass evaporation occurs. For a typical $\mathrm{Fe}_{70} \mathrm{Co}_{30}$ alloy sample as shown in Figures 4 and 5, the mole fraction of iron before and after the test series was measured to be 0.701 and 0.688 , respectively. Compositions before testing were determined gravimetrically; after testing, the samples were sent out to Luvak Laboratories for analysis using inert gas fusion ASTM E 1019-11 for oxygen concentration and direct current plasma emission spectroscopy ASTM E 1097-12 for metal concentrations.

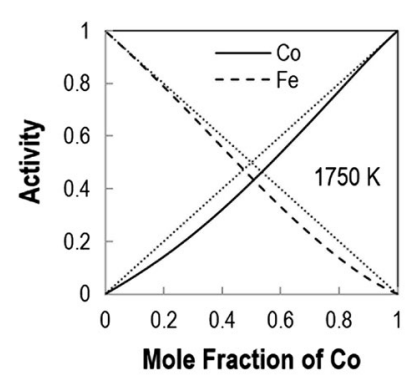

(a)

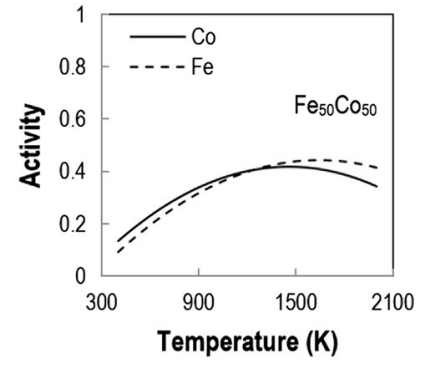

(b)
Fig. 3-Activity of iron-cobalt alloys as a function of composition at $1750 \mathrm{~K}\left(1476.85^{\circ} \mathrm{C}\right)(a)$ and activity of $\mathrm{Fe}_{50} \mathrm{Co}_{50}$ as a function of temperature $(b)$.

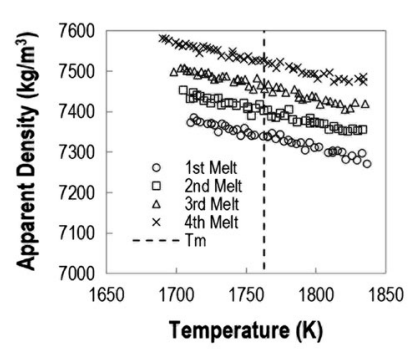

(a)

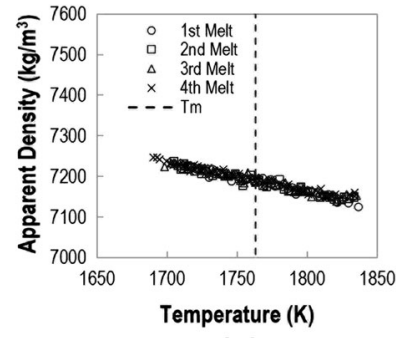

(b)
Fig. 4-Apparent density of the $\mathrm{Fe}_{70} \mathrm{Co}_{30}$ sample before (a) and after $(b)$ inclusion of a correction factor to account for mass evaporation.

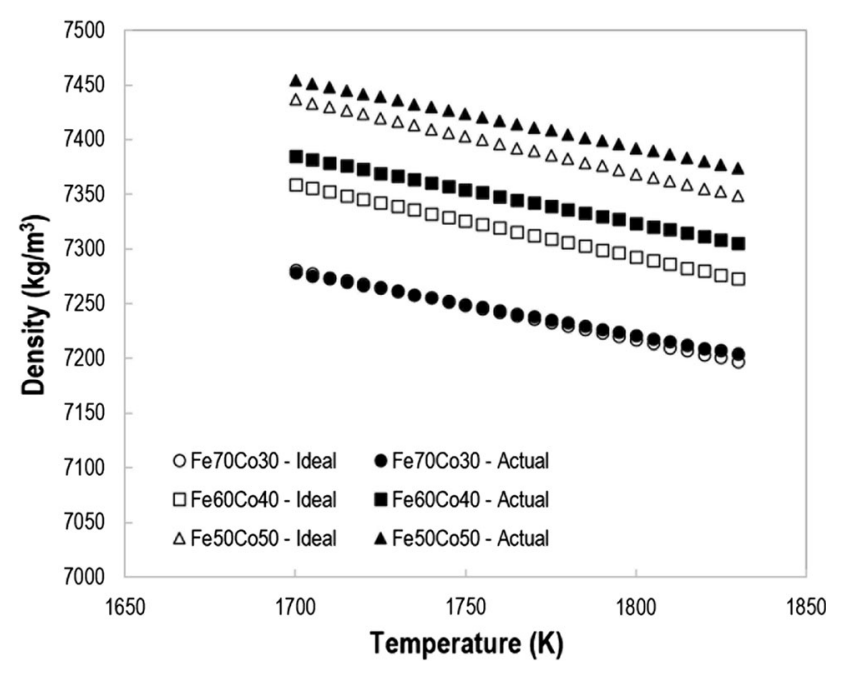

Fig. 5-Measured density and density of an ideal solution as a function of temperature. 
Using Eq. [1], mass evaporation was evaluated throughout each thermal cycle and the change in mass was tracked to allow calculation of density at any specific time $t$. The density for each melt cycle was thus estimated as a function of temperature in a range from the maximum superheating to the maximum undercooling temperatures (highlighted portion in Figure 2). The importance of tracking changes in mass is clearly illustrated in Figure 4. Mass evaporation was not included in the evaluation of apparent density in Figure 4(a). The combination of decreasing volume and assuming constant mass resulted in the increase and overestimation in apparent density as melt cycles accumulate. In contrast, by the proper consideration of mass evaporation, shown in Figure 4(b), a consistent density value was obtained independent of the test duration.

\section{RESULTS AND DISCUSSION}

The density of five different iron-cobalt alloy compositions were evaluated and fitted with linear functions; the density at its melting point in $\mathrm{K}\left(\rho_{T \mathrm{~m}}\right)$ and the slope (a) from Eq. [2] are also reported. Initially, three samples were prepared for each composition. One $\mathrm{Fe}_{60} \mathrm{Co}_{40}$ and one Co sample were lost during processing. The results of thirteen samples were listed in Table II.

$$
\rho(T)=\rho_{T_{\mathrm{m}}}+a\left(T-T_{\mathrm{m}}\right) .
$$

The density at the melting point increases as the mole fraction of cobalt increases; this is to be expected since cobalt is denser and the melting temperature is decreasing over this range. The coefficient of expansion (slope) also increases as the mole fraction of cobalt increases. In order to investigate deviation from ideal mixing behavior, Figure 5 compares the density for these alloys as a function of temperature assuming ideal mixing to the density calculated using the values given in Table II. Values vary by less than 0.45 pet at any given temperature.

To compare with previous work, the density at $1756 \mathrm{~K}\left(1482.85^{\circ} \mathrm{C}\right)$ was calculated using the fitted equations for each composition and plotted in Figure 6 with the density data measured by two different research groups. Watanabe et al. ${ }^{[8]}$ measured the density of seven different compositions using the MBP method. Brillo et $a l .^{[15]}$ utilized a ground-based EML facility to measure five different compositions. The two different non-contact methods yielded almost identical slope $(\sim 0.1$ pct $)$ with difference in density by up to 0.6 pct over all range of composition. Watanabe's data show $\sim 15$ pct difference in slope, and the difference in density value is up to 1.0 pct and 1.6 pct compared with Brillo and Lee, respectively. All three data sets show a good linear relationship between the density and composition indicating that the iron-cobalt system behaves almost like an ideal solution and has near zero excess volume. ${ }^{[15]}$ It is also confirmed from the Fe-Co equilibrium phase diagram that this system has very little solute segregation. ${ }^{[16]}$ The R-squared values for both EML and ESL are 0.993 and 0.998 , respectively, whereas data measured by MBP method show 0.978. In order to statistically compare the precision between the EML and ESL methods, the standard deviation of each data point from the fitted linear function was estimated for individual measurement.

Table III lists the composition, the number of data points, the standard deviation of each single measurement from a linear fit (zero excess volume), and the overall standard deviation for all data points from the linear fit. The $P$ value $(0.013)$ from the unpaired $t$ test with the 95 pct confidence interval confirmed that there was a statistically significant difference between the EML and ESL methods in terms of precision. The ESL measurement showed better precision giving the lower standard deviation by the factor of two. Notice that the variation of the standard deviation is also smaller for the ESL method, which implies better consistency. Using ESL processing technique generates superior results as compared to other ground-based measurement techniques. It remains to be shown if space-based EML processing will show similar improvements in precision.

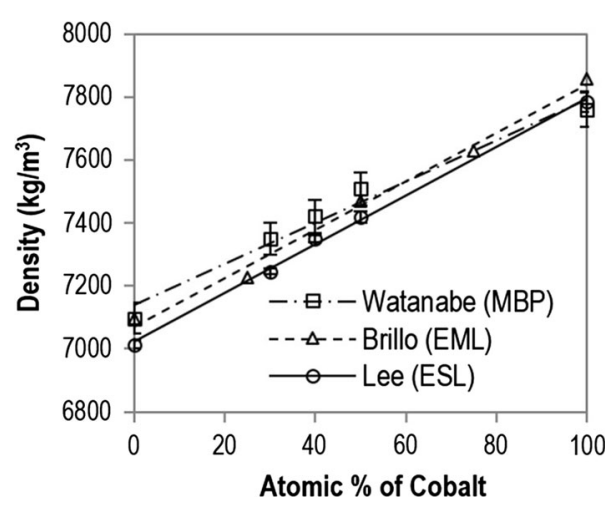

Fig. 6-Density of iron-cobalt alloy at $1756 \mathrm{~K}\left(1482.85^{\circ} \mathrm{C}\right)$.

Table II. Density Results of Tested Iron-Cobalt Alloy Samples

\begin{tabular}{lcccc}
\hline Composition (at.pct) & $T_{\mathrm{m}}(\mathrm{K})$ & Number of Samples & $\rho_{T \mathrm{~m}}\left(\mathrm{~kg} \mathrm{~m}^{-3}, \rho\right.$ at $\left.T_{\mathrm{m}}\right)$ & $a\left(\mathrm{~kg} \mathrm{~m} \mathrm{~K}^{-3} \mathrm{~K}^{-1}, \mathrm{~d} \rho / \mathrm{d} T\right)$ \\
\hline $\mathrm{Fe}$ & 1808 & 3 & 6983.74 & -0.581 \\
$\mathrm{Fe}_{70} \mathrm{Co}_{30}$ & 1763 & 3 & 7242.17 & -0.569 \\
$\mathrm{Fe}_{60} \mathrm{Co}_{40}$ & 1753 & 2 & 7352.53 & -0.612 \\
$\mathrm{Fe}_{50} \mathrm{Co}_{50}$ & 1750 & 3 & 7423.47 & -0.618 \\
$\mathrm{Co}$ & 1768 & 2 & 7774.30 & -0.779 \\
\hline
\end{tabular}


Table III. Density Results of Tested Iron-Cobalt Alloy Samples

\begin{tabular}{llcr}
\hline & Composition & Number of Data Points & Standard Deviation \\
\hline EML & Fe & 7 & 8.34 \\
& Fe75Co25 & 11 & 9.98 \\
Fe50Co50 & 16 & 12.43 \\
Fe25Co75 & 16 & 19.26 \\
Co & 15 & 12.35 \\
ESL & Overall deviation from linear fit & 5.37 \\
& Fe & 54 & 5.83 \\
& Fe70Co30 & 43 & 6.22 \\
& Fe60Co40 & 59 & 5.63 \\
& Fe50Co50 & 52 & 8.31 \\
& Co & 82 & 5.82 \\
& Overall deviation from linear fit & & 6.65 \\
\hline
\end{tabular}

\section{SUMMARY}

The density of the iron-cobalt system was measured using the ESL facility located at NASA MSFC. Five different compositions of the Fe-Co system were tested. The projected shape of the samples was captured with a high-speed camera and converted into volume data. The change in mass of the samples was estimated by Langmuir's equation including the effects of constituent activity, surface segregation, and potential changes in composition due to differential evaporation of each constituent. Finally, the density of the tested alloys was evaluated as a function of time and tabulated as a function of temperature using the estimated volume and mass taken from knowledge of the sample's thermal profile with time. The density results were compared with those measured by two different methods in the literature. The density measured by the ESL technique showed good agreement with those measured by the EML and the MBP methods but with enhanced precision. This research has demonstrated the applicability of the ESL technique to materials vulnerable to mass evaporation.

\section{ACKNOWLEDGMENTS}

The authors would like to thank Matthias Kolbe of DLR-Köln for help in performing measurements to confirm the lack of sedimentation in the Fe-Co system, as well as NASA MSFC ESL operators Trudy Allen and Glenn Fountain for their technical advice. This project is sponsored by NASA under grants NNX10AR71G and NNX14AB74G. Experimental portions of this research were performed at NASA Marshall Space Flight Center.

\section{REFERENCES}

1. K. Bornemann and P. Siebe: Z. Metallkd., 1922, vol. 14, pp. 329-34.

2. L. Wang and Q. Mei: J. Mater. Sci. Technol., 2006, vol. 22, pp. $1-3$.

3. A.H. Cook: Philos. Trans. R. Soc. A, 1961, vol. 254, pp. 125-54.

4. K.B. Khokonov, B.B. Alchigirov, and S.N. Zadumkin: Ind. Lab., 1974, vol. 40, pp. 56-57.

5. B.A. Mel'nik: Izv. AN. SSSR Mat., 1978, vol. 2, pp. 73-75.

6. G.H. Kaiura and J.M. Toguri: Can. Metall. Quart., 1979, vol. 18, pp. 155-64.

7. D.R. Gaskell, A. McLean, and R.G. Ward: Trans. Faraday Soc., 1969, vol. 65, pp. 1498-1508.

8. S. Watanabe and S. Tunezo: Trans. Jpn. Inst. Met., 1972, vol. 13, pp. 186-191.

9. I. Jimbo and A.W. Cramb: Metall. Trans. B, 1993, vol. 24B, pp. $5-10$

10. J. Brillo and I. Egry: Int. J. Thermophys., 2007, vol. 28, pp. 1004-16

11. M. Adachi, T. Aotagi, A. Mizuno, M. Watanabe, H. Kobatake, and H. Fukuyama: Int. J. Thermophys., 2008, vol. 29, pp. 2006-14.

12. Y. Inatomi, F. Onishi, K. Nagashio, and K. Kuribayashi: Int. J. Thermophys., 2007, vol. 28, pp. 44-59.

13. J. Lee and D.M. Matson: Int. J. Thermophys., 2014, vol. 35, pp. $1697-1704$.

14. T. Iida and R.I.L. Guthrie: The Physical Properties of Liquid Metals, Oxford University Press, Oxford, 1988, pp. 133-35.

15. J. Brillo, I. Egry, and T. Matsuchita: Int. J. Mater. Res., 2006, vol. 97 , pp. 1526-32.

16. J.E. Rodriguez and D.M. Matson: CALPHAD, 2015, vol. 49, pp. $87-100$. 